\title{
Methionine Coated with Various Materials Supplemented to Soybean Meal Diet for Fingerling Carp Cyprinus carpio and Channel Catfish Ictalurus punctatus
}

\author{
Takeshi Murai*1, Hiroshi Ogata*1, and Takeshi Nose*1 \\ (Received July 4, 1981)
}

\begin{abstract}
A six week feeding experiment was conducted to study effects of methionine coated with various materials added to soybean meal diet on growth and feed efficiency of fingerling carp and channel catfish. The coating materials used were aldehyde treated casein, acid treated casein, and hydrogenated tallow. Supplements of methionine, either coated or uncoated, significantly improved growth and feed efficiency of both species. In carp, addition of methionine coated with aldehyde treated casein significantly enhanced growth over that of uncoated methionine. But in channel catfish, coating methionine with any type of material did not significantly improve the utilization of supplemented methionine. Compared to the group fed the diet with uncoated methionine, addition of methionine coated with acid treated casein to the diet significantly depressed the growth of channel catfish. These results indicate that both carp and channel carfish can utilize crystalline methionine supplmented to soybean meal and that utilization of crystalline methionine by carp can be improved further by coating it with aldehyde treated casein.
\end{abstract}

Carp ${ }^{1)}$ and channel catfish ${ }^{27}$ have been reported to respond to amino acid diets only when $\mathrm{pH}$ of the diets was adjusted to higher than 5. However, the growth and feed efficiency of these fishes were still inferior to those fed an intact protein diet. Also, the supplements of crystalline amino acids to casein or soybean meal have been reported to show little effect on growth and feed efficiency of channel catfish..$^{3,4)}$ Recently, Robinson et al. ${ }^{6}$ have shown that fingerling channel catfish were able to utilize effectively feed grade lysine supplemented to a peanut meal diet.

In our previous study, ${ }^{\theta)}$ fingerling carp fed the gelatin diet supplemented with 10 amino acids coated individually with aldehyde treated casein showed more than four-fold faster growth and almost two-fold better feed efficiency than fish fed the gelatin diet supplemented with the same 10 amino acids, but uncoated. Addition of either the coated or uncoated arginine to casein also improved growth and feed efficiency without any significant difference between these two. Thus, the relative utilization of crystalline amino acids in these fishes is still contradictory and there might be differences in utilization of individual amino acid probably due to differences in a rate of intralumen absorption among free amino acids. ${ }^{7)}$

This experiment was carried out to study effects of supplemental methionine coated with various materials to soybean meal on growth and feed efficiency of carp and channel catfish fingerlings.

\section{Materials and Methods}

The composition and proximate analysis of experimental diets are presented in Table 1. The amino acid contents of the soybean meal (heat treated and defatted) ${ }^{* 2}$ used were determined after hydrolysis in $6 \mathrm{~N} \mathrm{HCl}$ at $110^{\circ} \mathrm{C}$ under vacuum. The estimation of tryptophan was performed by the method of Penke et al ${ }^{8)}$ using $3 \mathrm{~N}$ mercaptoethanesulfonic acid. Cystine was determined as cysteic acid after oxidizing the protein with performic acid according to a slight modification of the method of MOORE. ${ }^{9)}$ Analyses were carried out by an automatic amino acid analyzer (Hitachi Model 835) utilizing the high-resolution procedure. Analyzed values (diet 1) and amino acid profile of the diets are presented in Table 2.

Since amino acid requirements of carp and channel catfish are relatively similar as shown in Table 2 , the same diets were used for both fishes. The

*1 Division of Fish Nutrition, National Research Institute of Aquaculture, Hiruta, Tamaki, Watarai, Mie

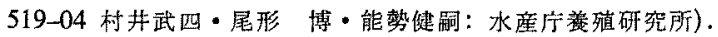

*2 Puffmin 10, product of Kikkoman Shoyu Co., Ltd. Analyzed nutrient contents were: Crude protein, $50.47 \%$; Crude fat, $1.54 \%$. 
Table 3. Effects of supplemental methionine to soybean meal on growth and feed efficiency (based on dry weight of each diet) of carp fingerlings*1

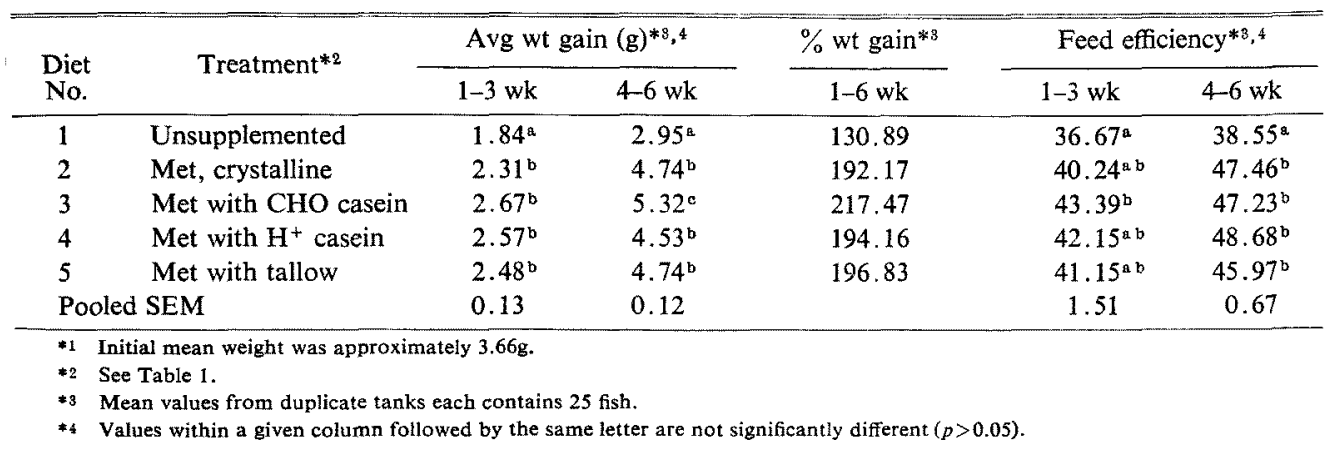

Table 4. Effects of supplemental methionine to soybean meal on growth and feed efficiency (based on dry weight of each diet) of channel catfish fingerlings*1

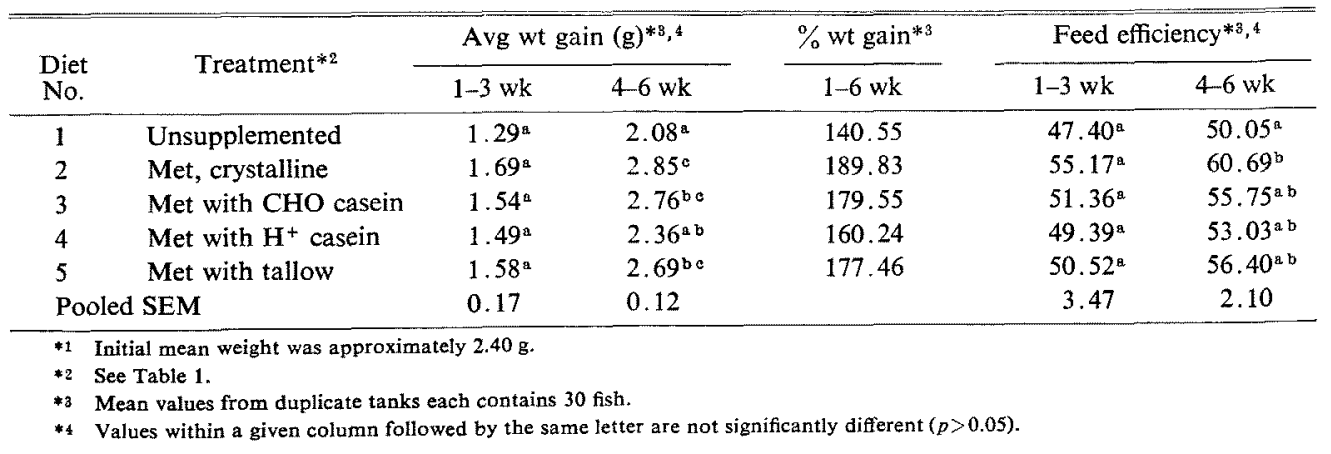

heated well water $\left(30 \pm 0.5^{\circ} \mathrm{C}\right)$. The remaining experimental conditions and statistical evaluation of the data employed were exactly the same as previously described. ${ }^{6)}$

\section{Results}

\section{Carp Study}

Carp fingerlings fed the diets supplemented with methionine either uncoated (diet 2) or coated (diet 3-5) showed significantly better weight gain than those fed the unsupplemented diet (diet 1) in 3 weeks (Table 3). No other significant difference in growth was achieved at this period. Differences in feed efficiency in this period were still marginal, but those data reflected the weight gain of each treatment.

During the second 3 week period, effect of any type of supplemental methionine on growth became much clearer than the first 3 week period and weight gain from the unsupplemented diet was less than $60 \%$ of the groups with supplemental methionine. In the groups with supplemental methionine, supplement of methionine coated with $\mathrm{CHO}$ casein (diet 3) improved weight gain further and resulted in the best growth performance which was more than $200 \%$ weight gain in 6 weeks. While supplement of methionine coated with $\mathrm{H}^{+}$casein (diet 4) or tallow (diet 5) did not improve growth performance over that of uncoated methionine. Supplementation of any type of methionine used in this study significantly improved feed efficiency also, but differences among dietary groups supplemented with methionine were fractional and no clear correlationship between growth and feed efficiency was noted.

\section{Channel Catfish Study}

In the first 3 week period, effects of supplemental methionine either coated or uncoated on growth and feed efficiency did not become significant. However, channel catfish fingerlings fed the diets supplemented with any type of methionine (diet 2-5) showed slightly better growth performance than those fed the unsupplemented diet (diet 1) and feed efficiency data reflected the weight 
gain of each treatment (Table 4).

During the second 3 week period, the trend in growth response to the treatments was exactly the same as the previous period, but weight gain of fish fed the diets with uncoated methionine (diet 2$)$ and coated with CHO casein (diet 3) or tallow (diet 5) became significantly better than that fed the unsupplemented diet. While supplement of methionine coated with $\mathrm{H}^{+}$casein (diet 4) failed to improve weight gain significantly. The best weight gain which was almost $190 \%$ in 6 weeks was attained by feeding the diet with uncoated methionine. This weight gain was also slightly better than those from diets 3 and 5 . Feed efficiency data in this period again reflected the weight gain of each treatment, but only group showed significantly better feed efficiency than the unsupplemented group was the one with uncoated methionine.

\section{Discussion}

Data from this study clearly indicate that both carp and channel catfish can efficiently utilize crystalline methionine supplemented to soybean meal, in spite of the fact that the level of total sulfur amino acids in the unsupplemented diet was higher than reported requirement of channel carfish. ${ }^{12}$ ) ANDREWS and $\mathrm{PAGE}^{4}$ ) have reported that addition of crystalline methionine or cystine to soybean meal did not significantly improve growth of channel catfish. The discrepancy between these two studies may be due to the difference in the level of total sulfur amino acids in the basal diets, because their diet contained at least $20 \%$ higher level of those than ours. While, it has been reported that additions of amino acids to soybean meal simulating those levels of essential amino acids in trout eggs improved growth of rainbow trout, but supplement of methionine alone had no effect. ${ }^{13}$ ) Thus, not only the level of individual amino acid, but balance of amino acids also may affect the utilization of crystalline amino acids.

The growth data of carp in this study indicate that the differences in coating materials may affect utilization of crystalline methionine. Exact mechanism which caused this difference is still unknown, but utilization of crystalline methionine can be improved further by coating it with aldehyde treated casein. Whereas, the fact that the unsupplemented diet contained methionine higher than the channel catfish's requirement ${ }^{12)}$ may partly be responsible for no significant improvement of methionine utilization by coating with any of materials used in this study.

In order to improve nutritional value of certain ingredients, such as soybean meal by supplementing crystalline amino acids for carp or channel catish, more informations on mechanisms of amino acids absorption in these fishes are needed. Nevertheless, coating amino acids with casein may be one of the promising tools at least for carp.

\section{References}

1) T. Nose, S. ARai, D. LeE, and Y. Hashimoto: Bull. Japan. Soc. Sct. Fish., 40, 903-908 (1974).

2) R. P. WILSON, D. E. HARdING, and D. L. GARLING: J. Nutr., 107, 166-170 (1977).

3) J. W. ANDrews, J. W. PAGe, and M. W. MURRAY: J. Nutr., 107, 1153-1156 (1977).

4) J.W. Andrews and J. W. PAGE: J. Nutr., 104, 1091-1096 (1974).

5) E. H. Robinson, R. P. Wilson, and W. E. POE: J. Nutr., 110, 2313-2316 (1980).

6) T. Mural, T. Akiyama, and T. Nose: Bull. Japan. Soc. Sci. Fish. 47, 523-527 (1981).

7) D. B. A. Silk, T. C. Marrs, J. M. Addison, D. Burston, M. L. Clark, and D. M. Matthews: Clin. Sci. Mol. Med., 45, 715-719 (1973).

8) B. Penke, R. Ferenczi, and K. Kovacs: Anal. Biochem., 60, 45-50 (1974).

9) S. Moore: J. Biol. Chem., 238, 235-237 (1963).

10) T. Nose: Proc. World Symp, on Finfish Nutrition and Fishfeed Technology, 1. 145-156 (1979).

11) E. H. Robinson, W. E. Poe, and R. P. Whlson: Feedstuffs, 52, 29 and 36 (1980).

12) D. E. Harding, O. W. Allen, and R. P. Wilson: J. Nutr, 107, 2031-2035 (1977).

13) G. L. Rumsex and H. G. KeTOLA: J. Fish. Res. Board Can., 32, 422-426 (1975). 